\title{
Berberine Moderates Glucose and Lipid Metabolism through Multipathway Mechanism
}

\author{
Qian Zhang, Xinhua Xiao, Kai Feng, Tong Wang, Wenhui Li, Tao Yuan, Xiaofang Sun, Qi Sun, \\ Hongding Xiang, and Heng Wang
}

Department of Endocrinology, Peking Union Medical College Hospital, Chinese Academy of Medical Sciences, Beijing 100730, China

Correspondence should be addressed to Xinhua Xiao, xiaoxinhua@medmail.com.cn and Kai Feng, fengkai@medmail.com.cn

Received 7 May 2010; Accepted 21 August 2010

Copyright ( 2011 Qian Zhang et al. This is an open access article distributed under the Creative Commons Attribution License, which permits unrestricted use, distribution, and reproduction in any medium, provided the original work is properly cited.

\begin{abstract}
Berberine is known to improve glucose and lipid metabolism disorders, but the mechanism is still under investigation. In this paper, we explored the effects of berberine on the weight, glucose levels, lipid metabolism, and serum insulin of KKAy mice and investigated its possible glucose and lipid-regulating mechanism. We randomly divided KKAy mice into two groups: berberine group (treated with $250 \mathrm{mg} / \mathrm{kg} / \mathrm{d}$ berberine) and control group. Fasting blood glucose (FBG), weight, total cholesterol (TC), triglyceride (TG), high-density lipoprotein-cholesterol (HDL-c), low-density lipoprotein-cholesterol (LDL-c), and fasting serum insulin were measured in both groups. The oral glucose tolerance test (OGTT) was performed. RT ${ }^{2}$ PCR array gene expression analysis was performed using skeletal muscle of KKAy mice. Our data demonstrated that berberine significantly decreased FBG, area under the curve (AUC), fasting serum insulin (FINS), homeostasis model assessment insulin resistance (HOMA-IR) index, TC, and TG, compared with those of control group. RT ${ }^{2}$ profiler PCR array analysis showed that berberine upregulated the expression of glucose transporter 4 (GLUT4), mitogen-activated protein kinase 14 (MAPK14), MAPK8(c-jun N-terminal kinase, JNK), peroxisome proliferator-activated receptor $\alpha$ (PPAR $\alpha$ ), uncoupling protein 2 (UCP2), and hepatic nuclear factor $4 \alpha(\mathrm{HNF} 4 \alpha)$, whereas it downregulated the expression of PPAR $\gamma$, CCAAT/enhancer-binding protein (CEBP), PPAR $\gamma$ coactivator $1 \alpha$ (PGC $1 \alpha)$, and resistin. These results suggest that berberine moderates glucose and lipid metabolism through a multipathway mechanism that includes AMP-activated protein kinase-(AMPK-) p38 MAPK-GLUT4, JNK pathway, and PPAR $\alpha$ pathway.
\end{abstract}

\section{Introduction}

Type 2 diabetes mellitus (T2DM) is a metabolic disorder characterized by dysregulation of carbohydrate, protein, and fat metabolism resulting from defects in insulin secretion, insulin action, or both [1]. The number of T2DM patients is expected to rise to 300 million worldwide by the year 2025 due to an increased number of elderly people, a greater prevalence of obesity, and sedentary lifestyles [2]. Besides hyperglycemia, several other symptoms, including hyperlipidemia, are involved in the development of microvascular and macrovascular complications of diabetes, which are the major causes of morbidity and death. Therefore, it is especially important to reinforce effective prevention and regular treatment of this disease. However, since patient compliance with diet and exercise regiments is often poor and medications are needed and because many oral medications have a number of serious adverse effects, management of hyperglycemia or hyperlipidemia with low side effects remains a challenge to the medical system. Traditional Chinese medicines and their extractions demonstrate the characteristics of economy and effectiveness in managing diabetes and its complications.

Rhizoma Coptidis was recorded as an antidiabetes medication about 1500 years ago in a book titled "Note of Elite Physicians" by Hongjing Tao. Berberine is the major active component of Rhizoma coptidis. Recent studies have demonstrated beneficial effects of berberine on metabolism disorders including weight control, cholesterol reduction, antilipogenic and hypoglycemic effects, and even inhibiting chronic cocanine-induced sensitization [3-6].

Many studies have been published on the glucosereducing mechanism of berberine. Zhou et al. found that berberine stimulated glucose transport through a mechanism distinct from insulin in 3T3-L1 adipocyes [7]. Moreover, berberine could activate AMPK and induced glycolysis in L6, C2C12, and 3T3-L1 cell lines [8]. And berberine dose-dependently inhibited respiration in L6 myotubes by 
its specific effect on respiratory complex I [9]. Regarding the mechanism of berberine in moderating lipid metabolism, Lee et al. found that berberine moderated lipids by inhibiting adipogenesis in 3T3-L1 adipocytes [10]. Two trials revealed that berberine activated extracellular signal-regulated kinase (ERK) [7] and JNK [11] in HepG2 cells. Given these results, we hypothesize that berberine may exhibit a multitargeted mechanism in moderating glucose and lipids.

Trial materials used in biomedical studies often involve cells. However, in this study, KKAy mice were used to investigate the effects of berberine on glucose and lipid metabolism in vivo. KKAy mice are developed by transferring the yellow obesity (Ay) gene into the KK strain, which show severe obesity, hyperglycemia, hyperinsulinemia, and glucose intolerance by eight weeks of age. So, they are especially useful for evaluating of antidiabetic and antiobesity agents. The skeletal muscle plays a major role in energy balance. It accounts for $>30 \%$ of energy expenditure and is the primary tissue of insulin stimulating glucose uptake, disposal, and storage [12]. To understand the mechanism that berberine regulates glucose and lipids, we performed $\mathrm{RT}^{2} \mathrm{PCR}$ diabetes superarray to analyze the expression of diabetes-related genes in skeletal muscle tissue of KKAy mice. Natural products are gaining increased applications in drug discovery and development. Being chemically diverse, they are able to modulate several targets simultaneously in a complex system. DNA microarrays serve as suitable high-throughput tool for simultaneous analysis of multiple genes [13].

\section{Materials and Methods}

2.1. Animal Modeling, Grouping, and Treatment. Male KKAy mice (from the Chinese Academy of Medical Sciences, Beijing, China) were fed in the standard mouse-feeding room. The mice were fed with high-fat laboratory chow (fat: carbohydrate: protein $=58: 25.6: 16.4$ ). All procedures were approved by the Ethics Committee for the Use of Experimental Animals of Peking Union Medical College Hospital. Before drug administration, murine blood samples for blood glucose measurement were collected from the tail vein. KKAy mice with random blood glucose values above $11.1 \mathrm{mmol} / \mathrm{L}$ were considered diabetic. These mice were randomly divided into two groups: berberine group $(n=8$, ig $250 \mathrm{mg} \cdot \mathrm{kg}^{-1} \cdot \mathrm{d}^{-1}$ berberine) and control group ( $n=8$, ig the same volume of normal saline). Drugs were given to the mice between 8:00 and 9:00 AM every day. Over a four-week period, on days 0 (before treatment), 7, 14, 21, and 28, weight and FBG of the KKAy mice (4-hour fast) were measured in blood samples obtained from tail veins. An oral glucose tolerance test (OGTT) was performed on day 21 . On day 28, blood samples of the KKAy mice were again taken for measuring FINS and lipid metabolic parameters, after anesthesia. The mice were then sacrificed and their skeletal muscles were collected and stored in dry ice.

2.2. Oral Glucose Tolerance Test (OGTT). After the mice fasted for 4 hours, glucose $2.2 \mathrm{~g} / \mathrm{kg}$ was orally administered. Then blood samples were collected from tail veins at 0 (prior to glucose load), 30, 60, and 120 minutes (after glucose load) for the glucose assay. AUC was calculated for blood glucose (BG) during the OGTT: $\mathrm{AUC}=0.5 \times[\mathrm{Bg} 0+\mathrm{Bg} 30] / 2+0.5$ $\times[\mathrm{Bg} 30+\mathrm{Bg} 60] / 2+1 \times[\mathrm{Bg} 60+\mathrm{Bg} 120] / 2$.

2.3. Measurement of Serum Parameters. Blood glucose was measured by the glucose oxidase peroxidase (Bayer Breeze blood glucose meter, Germany) method. TC, TG, LDL-c, and HDL-c were assayed by enzyme end-point method (Roche, Germany). Serum insulin was measured by enzymelinked immunosorbent assay (ELISA) using rat/mice insulin ELISA kit (LINCO Research, USA). HOMA-IR index was calculated according to the following formula: HOMA-IR = FBG $(\mathrm{mmol} / \mathrm{L}) \times$ FINS $(\mu \mathrm{U} / \mathrm{mL}) / 22.5$.

\section{4. $R T^{2}$ Profiler PCR Array}

2.4.1. First Strand cDNA Synthesis. Total RNA was extracted from the skeletal muscle of 3 mice from the berberine group and 3 mice from the control group, using TRIZOL Reagent (Invitrogen Life Technologies, USA). RNA cleanup used RNeasy MinElute Cleanup Kit (Qiagen, Germany). RNA quality was determined by running a sample with RNA loading dye (Ambion, USA) on a $1 \%$ agarose gel and inspecting for distinct $18 \mathrm{~S}$ and $28 \mathrm{~S}$ bands, indicating lack of degradation. Quantity was determined by A260 and A280 measurement. All samples had A260/A280 ratios of 1.9-2.1. SuperScript Reverse Transcriptase was applied to reversetranscribe RNA into first-strand cDNA. To analyze the differential expression of multiple genes involved in diabetes mellitus, we used $\mathrm{RT}^{2}$ profiler PCR mouse diabetes mellitusspecific expression arrays (SuperArray, Frederick, MA, USA), which uses SYBR Green-based real-time PCR to assay a large number of genes simultaneously. Each superarray membrane contained 84 specific cDNA fragments of genes involved in diabetes mellitus, including receptors, transporters and channels, nuclear receptors, metabolic enzymes, secreted factors, transcription factors, and others. Table 1 lists the genes measured in this study. We added cDNA to each well of an $\mathrm{RT}^{2}$ profiler PCR diabetes array for quantitative PCR in the ABI PRISM 7700 system (Applied Biosystems, USA) with the following cycling conditions: an initial denaturation at $95^{\circ} \mathrm{C}$ 15 minutes, and 40 cycles of $95^{\circ} \mathrm{C} 15$ seconds, $55^{\circ} \mathrm{C} 60 \mathrm{sec}-$ onds, with a final infinite $4^{\circ} \mathrm{C}$ hold. Fluorescence signal was then collected. For quality control purpose, no reverse transcription control and no template control were performed.

2.4.2. Data Normalization and Analysis. Five endogenous control genes - glucuronidase $\beta$ (GUS $\beta$ ), hypoxanthine guanine (HPRT1), heat-shock protein (HSP90), glyceralsehyde(GAPDH), and $\beta$-actin(ACT $\beta)$-present on the PCR Array were used for normalization. Each replicate cycle threshold $(\mathrm{Ct})$ was normalized to the average $\mathrm{Ct}$ of 5 endogenous controls on a per plate basis. The comparative Ct method was used to calculate the relative quantification of gene expression. The following formula was used to calculate the relative amount of the transcripts in the berberinetreated samples and samples of the control group, both of 
TABLE 1: Gene list of $\mathrm{RT}^{2}$ profiler PCR mouse diabetes array.

\begin{tabular}{lccccccccccc}
\hline Ace & Acly & Adra1a & Adrb3 & Agt & Akt2 & Aqp2 & Ccl5 & Ccr2 & Cd28 & Ceacam1 & Cebpa \\
Ctla4 & Dpp4 & Dusp4 & Enpp1 & Fbp1 & Foxc2 & Foxg1 & Foxp3 & G6pc & G6pd2 & Gcg & Gcgr \\
Glp1r & Gpd1 & Gsk3b & Hmox1 & Hnf4a & Icam1 & Ide & Ifng & Igfbp5 & Ikbkb & Il10 & Il12b \\
Il4ra & Il6 & Inpp11 & Ins1 & Pdx1 & Irs1 & Mapk14 & Mapk8 & Neurod1 & Nfkb1 & Nos3 & Nrf1 \\
Nsf & Parp1 & Pax4 & Pck1 & Pfkfb3 & Pik3cd & Pik3r1 & Ppara & Pparg & Ppargc1a & Ptpn1 & Pygl \\
Rab4a & Retn & Sell & Serpine1 & Slc14a2 & Slc2a4 & Snap23 & Snap25 & Sod2 & Srebf1 & Stx4a & Stxbp1 \\
Stxbp4 & Hnf1b & Tgfb1 & Tnf & Tnfrsf1a & Tnfrsf1b & Trib3 & Ucp2 & Vamp2 & Vamp3 & Vapa & Vegfa \\
Gusb & Hprt1 & Hsp90ab1 & Gapdh & Actb & MGDC & RTC & RTC & RTC & PPC & PPC & PPC \\
\hline
\end{tabular}

TABLE 2: Effect of berberine on the body weight and FBG of KKAy mice.

\begin{tabular}{|c|c|c|c|c|c|c|}
\hline Group & $n$ & Day 0 & Day 7 & Day 14 & Day 21 & Day 28 \\
\hline \multicolumn{7}{|l|}{ Control } \\
\hline Weight (g) & 8 & $30.51 \pm 2.44$ & $31.92 \pm 3.28$ & $32.21 \pm 3.15$ & $32.90 \pm 1.34$ & $33.04 \pm 1.42$ \\
\hline $\mathrm{FBG}(\mathrm{mmol} / \mathrm{L})$ & 8 & $16.15 \pm 8.98$ & $19.60 \pm 2.15$ & $18.28 \pm 5.05$ & $24.74 \pm 8.89$ & $20.54 \pm 5.85$ \\
\hline \multicolumn{7}{|l|}{ Berberine } \\
\hline Weight (g) & 8 & $30.08 \pm 1.94$ & $30.86 \pm 1.08$ & $31.04 \pm 1.76$ & $31.55 \pm 1.17$ & $31.88 \pm 0.74$ \\
\hline $\mathrm{FBG}(\mathrm{mmol} / \mathrm{L})$ & 8 & $16.45 \pm 8.04$ & $9.90 \pm 5.69^{*}$ & $10.35 \pm 4.14^{*}$ & $10.20 \pm 2.48^{* *}$ & $9.90 \pm 2.95^{* *}$ \\
\hline
\end{tabular}

Values are means \pm SD.

*Indicates significantly different versus control $(P<.05)$; ** $(P<.01)$.

which were normalized to the endogenous controls. $\Delta \Delta \mathrm{Ct}$ $=\Delta \mathrm{Ct}$ (berberine group) $-\Delta \mathrm{Ct}$ (control group) for RNA samples [14]. $\Delta \mathrm{Ct}$ is the $\log 2$ difference in $\mathrm{Ct}$ between the target gene and endogenous controls abstained by subtracting the average $\mathrm{Ct}$ of controls from each replicate. The fold change for each berberine-treated sample relative to the control sample $=2^{-\Delta \Delta \mathrm{Ct}}$.

2.4.3. Sensitivity Detection and Identification Expressed Genes. PCR Array quantification was based on the $\mathrm{Ct}$ number. $\mathrm{Ct}$ was defined as 35 for the $\Delta \mathrm{Ct}$ calculation when the signal was under detectable limits. A list of differentially expressed genes was identified using a 2 -tailed $t$-test. Changes in gene expression between the berberine group and the control group were illustrated as a fold increase/decrease. The criteria were a $P$ value $<.05$ and a mean difference equal to or greater than 2 -fold. The statistical calculation was based on the webbased program of $\mathrm{RT}^{2}$ profiler PCR Array Data Analysis. Genes that suited the above criteria were considered to be up- or downregulated. The experiments were repeated three times.

2.5. Statistical Analysis. All results are expressed as means \pm SD. The statistical value of $P<.05$ was considered as statistical significance. Differences between the berberine group and the control group were determined using the Mann-Whitey $U$ test. All statistical analyses were performed with SPSS version 12.0 software.

\section{Results}

3.1. Berberine Showed no Effect on Body Weight of KKAy Mice. No significant differences were noted between the initial body weight of KKAy mice in the berberine group and the control group. Also, after treatment, no significant differences were found between groups, suggesting that berberine does not affect body weight (Table 2, Figure 1(a)).

3.2. Berberine Decreased FBG of KKAy Mice. Before drug administration, FBG in both groups was similar. However, the FBG in the berberine group displayed a significant decrease on day $7(P<.05)$, day $14(P<.05)$, day 21 $(P<.01)$, and day $28(P<.01)$ (Table 2 , Figure $1(\mathrm{~b}))$. This showed that administration of berberine reduced the FBG of KKAy mice.

3.3. Berberine Improved the Glucose Tolerance of KKAy Mice. Blood glucose levels in two groups became elevated within 30 minutes after the administration of glucose to mice. Blood glucose levels recovered to the original levels after 120 minutes. The blood glucose level in mice treated with berberine was significantly suppressed at 0 minutes $(P<$ $.01)$, 30 minutes $(P<.01), 60$ minutes $(P<.05)$, and 120 minutes $(P<.05)$ compared to those in the control group (Table 3, Figure 2). AUC in the berberine group was significantly smaller than that in the control group $(P<$ $.05)$ (Table 3, Figure 3(a)). This indicated that berberine improved the glucose tolerance of KKAy mice.

3.4. Berberine Reduced FINS and HOMA-IR in KKAy Mice. The mice fed with berberine exhibited significantly lower fasting serum insulin levels than the control group $(P<.05)$ (Table 4, Figure 3(b)). The HOMA-IR index of the berberine group was $30 \%$ of that of the control group $(P<.05)$ (Table 4, Figure 3(c)). These data showed that berberine ameliorated the insulin sensitivity of KKAy mice. 


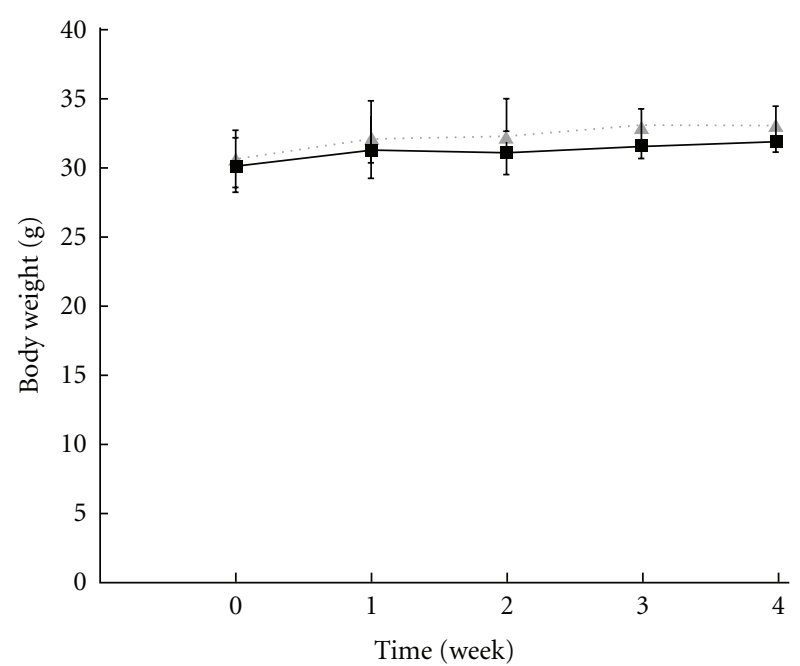

-.... Control

$\rightarrow$ Berberine

(a)

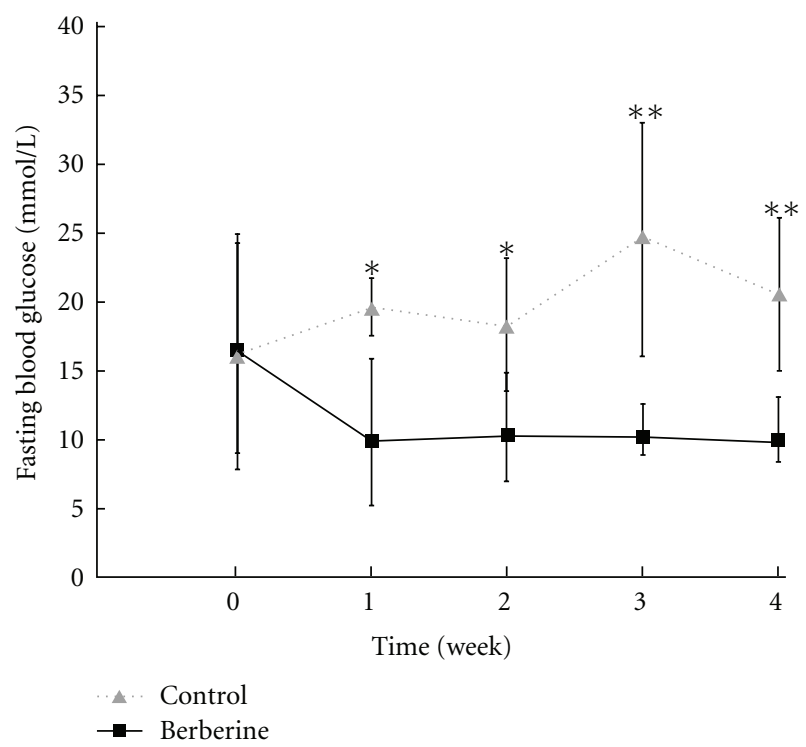

(b)

FIGURE 1: Weekly variation of body weight (a) and fasting blood glucose (b) between berberine group and control group. Data are expressed as means $\pm \mathrm{SD}$. ${ }^{*} P<.05,{ }^{* *} P<.01$ compared with that in the control.

3.5. Berberine Declined TC and TG in KKAy Mice. In addition to its glucose-lowering effects, berberine may have lipidregulating activity. After 4 weeks of treatment, the animals fed with berberine exhibited a significant decrease in TC $(P<$ $.05)$ and TG level $(P<.05)$. However, differences in HDL$c$ and LDL-c between the two groups were not statistically significant (Table 4, Figure 3(d)).

3.6. $R T^{2}$ Profiler PCR Array. Based on the results of the $\mathrm{RT}^{2}$ profiler PCR array, we found no significantly different genes expression between berberine and control group related to the insulin signaling pathway. For example, gene expression

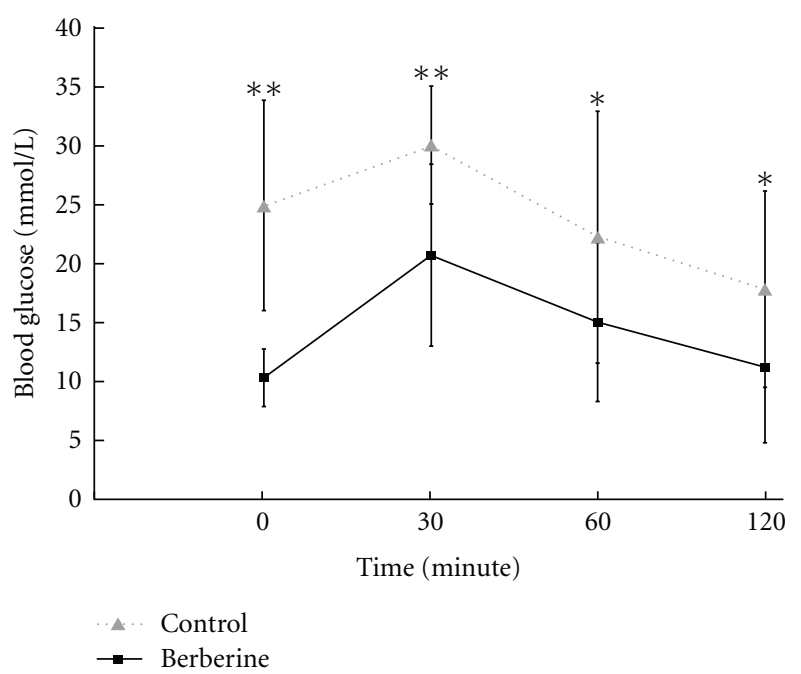

FIGURE 2: Comparison of plasma glucose concentration responses to an oral glucose tolerance test between berberine group and control group. Data are expressed as means $\pm \mathrm{SD} .{ }^{*} P<.05$, ${ }^{* *} P<.01$ compared with that in the control.

of thymoma viral proto-oncogene 2(AKT2), inhibitor of kappaB kinase $\beta$ (IKBKB), insulin receptor substrate (IRS-1), phosphatidlyinositol-3 kinase regulatory subunit polypeptide 1 (PIK3R1), phosphatidylinositol 3-kinase catalytic $\delta$ polypeptide (PIK3CD), and protein tyrosine phosphatase nonreceptor type 1(PTPN1,PTP1B), all of which are involved in the insulin signaling pathway, were not markedly upregulated or downregulated.

Based on gene selection criteria $(P<.05$ and fold change $\geq 2$ ), 10 genes that were $\geq 2$-fold upregulated or downregulated in the berberine group as shown in Table 5 and Figure 4.

According to the gene expression profile, we found that berberine could upregulated the expression of 2 genes related to the MAPK (mitogen-activated protein kinase) pathway. For example, gene expression of MAPK8 and MAPK14, both of which are involved in the MAPK pathway, was upregulated (13.15-fold and 4.17-fold, resp.) in the berberine group. Also, glucose transporter 4 (GLUT4) gene expression, which is involved in glucose transport in membranes, was upregulated by 4.48 -fold in the berberine group. Gene expression of peroxisome proliferator-activated receptor $\gamma$

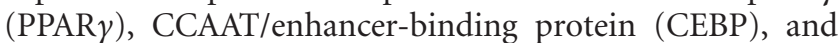
PPAR $\gamma$ coactivator $1 \alpha$ (PGC), which play important roles in adipogenesis, were downregulated (5.51-fold, 3.06-fold, and 2.70 -fold, resp.). PPAR $\alpha$ gene expression, which leads to reduction of plasma TG levels, was upregulated by 2.10 fold in the berberine group. In addition, gene expression of uncoupling protein 2 (UCP2), whose expression is involved in energy balance, was upregulated by 2.55 -fold in the berberine group. Furthermore, expression of hepatic nuclear factor $4 \alpha$ (HNF- $4 \alpha$ ) gene, a key regulator of glucose, cholesterol, and fatty acid metabolism, was upregulated by 2.12-fold in the berberine group. Gene expression of resistin, which contributes to insulin resistance, was downregulated by 2.12 -fold in the berberine group. 


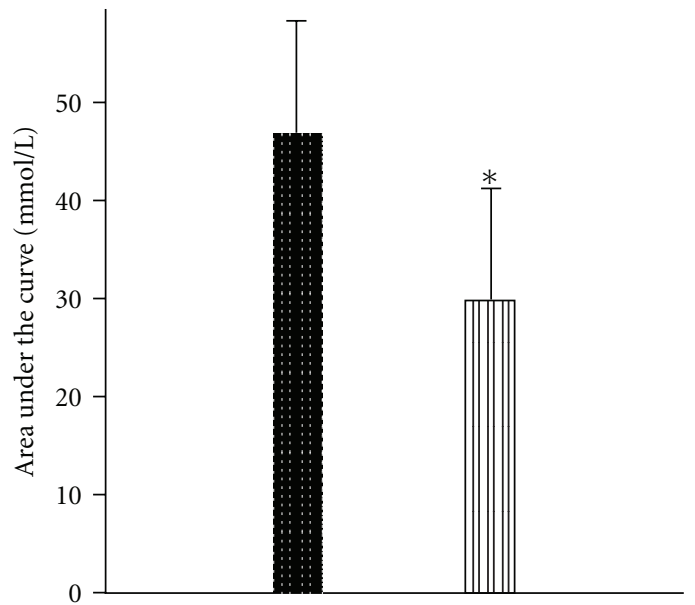

(a)

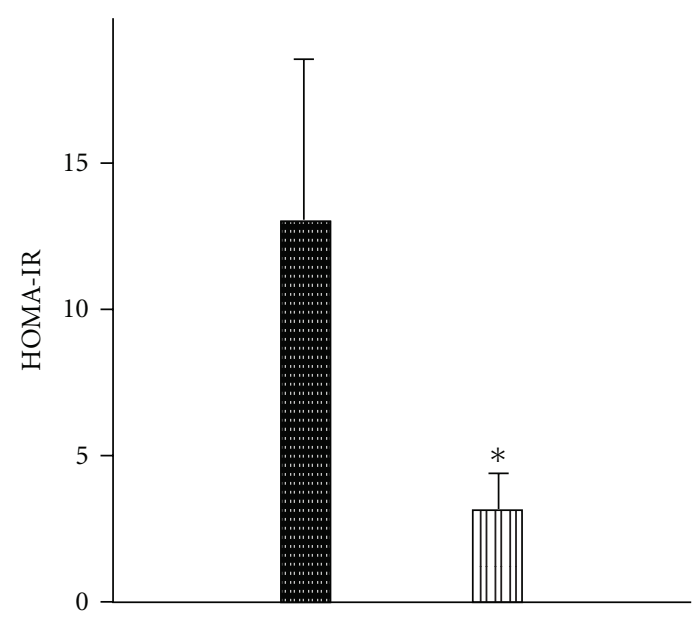

Control

سl] Berberine

(c)

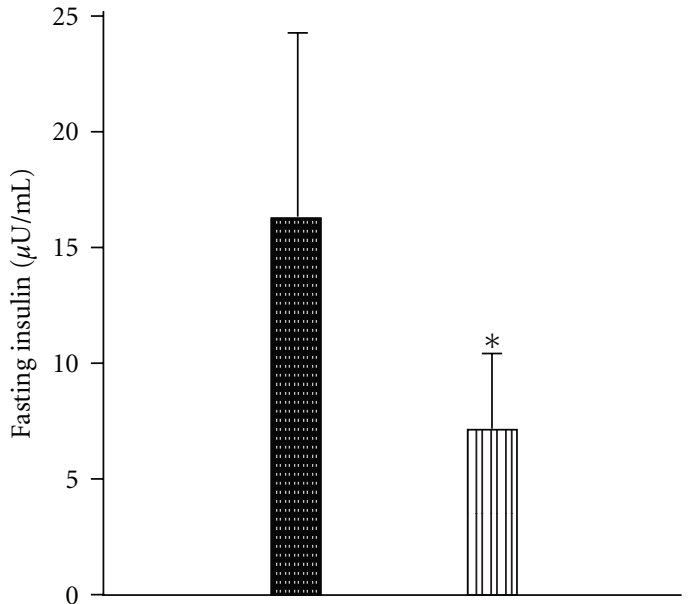

(b)

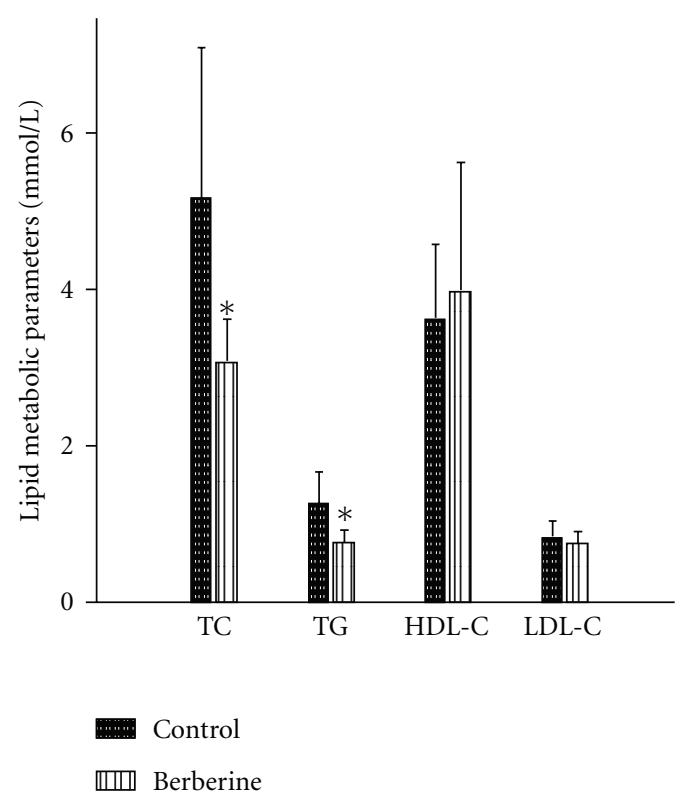

(d)

FIGURE 3: Area under the curve in OGTT trial (a), serum concentration of fasting insulin (b), HOMA-IR, (c) and lipid metabolic parameters (d) between berberine group and control group. Data are expressed as means $\pm \mathrm{SD} .{ }^{*} P<.05$ compared with that in the control.

\section{Discussion}

In this study, we found that the administration of berberine to KKAy mice significantly reduced FBG levels, FINS, and HOMA-IR index. Our results suggest that berberine has glucose-moderating action and can ameliorate oral glucose tolerance and insulin sensitivity. This result is similar to the results of other studies [7, 8, 10, 15-17]. However, Lee et al. treated ob/ob mice with berberine for 25 days and found that berberine could reduce the body weight [10]. In our study, berberine did not reduce body weight significantly. Othermore, berberine decreased serum TG and TC levels in KKAy mice. Two groups revealed the similar results $[18,19]$. However, Kong et al. found that oral administration of berberine in 32 hypercholesterolemic patients for 3 months reduced LDL-cholesterol by $25 \%$ [19]. While in this study, we found no evidence that berberine reduces serum LDL-c.

The mechanism of the hypoglycemic action of berberine is still under investigation. Our gene expression profile results showed no significant differences in gene expression between the berberine and the control group related to the insulin signaling pathway, including AKT2, IKBKB, IRS1, PIK3R1, PIK3CD, and PTPN1 (PTP1B). In the insulin signaling pathway, insulin stimulates glucose uptake to muscle and fat tissue [20]. It involves certain signal molecules, such as insulin receptor (IR), IRS-1, phosphatidylinositol 3kinase $(\mathrm{PI} 3 \mathrm{~K})$, and AKT. Defects are found in the insulin signaling pathway in muscle tissue of T2DM. Based on our 
results, we do not consider the insulin signaling pathway to be the key mechanism of the glucose-moderating effect of berberine. Other research supports this view. Berberine only weakly stimulated the phosphorylation of AKT/PKB [21] and did not augment tyrosine phosphorylation of IR and IRS-1 in 3T3-L1 cells [22]. Unlike insulin, the effect of berberine on increasing glucose uptake was insensitive to wortmannin, an inhibitor of PI3K, and SB203580, an inhibitor of p38 MAPK [7]. IKBKB can phosphorylate IRS1 on Ser307, thereby rendering IRS1 less amenable to become phosphorylated on tyrosine residues required to propagate the signal to downstream effectors such as protein kinase B [23]. PTP1B may dephosphorylate IRS-1 [24, 25]. Other studies suggest that PTP1B polymorphisms may be associated with obesity and insulin resistance in humans [2628]. The overexpression of PTP1B in muscle tissue results in insulin resistance [29].

We disclosed that berberine could upregulate the expression of 2 genes related to MAPK (mitogen-activated protein kinase) pathway and GLUT4. This suggests that berberine can activates MAPK in KKAy mice. The p38 MAPK cascade is downstream of AMPK in the signaling pathway of AICARstimulated glucose transport in Clone 9 cells $[30,31]$. In muscle tissue, p38 MAPK takes part in the activation of GLUT4 [30, 32]. It is possible that berberine can ameliorate glucose uptake in T2DM mice through the AMPKp38 MAPK-GLUT4 pathway. The possibility that berberine activate MAPK is still under investigation. Cheng et al. found that berberine strongly promotes the phosphorylation of AMPK and p38 MAPK on L6 rat skeletal muscles [21]. Moreover, berberine-stimulated glucose uptake was inhibited by the AMPK inhibitor Compound $\mathrm{C}$ and the p38 MAPK inhibitor SB202190. Inhibition of AMPK reduced p38 MAPK phosphorylation, suggesting that AMPK lies upstream of p38 MAPK. These results suggest that berberine circumvents insulin signaling pathways and stimulates glucose uptake through the AMP-AMPK-p38 MAPK pathway, which may account for the antihyperglycemic effects of this drug [21]. Zhou et al. found that in isolated rat skeletal muscles, metformin stimulates glucose uptake coincident with AMPK activation [33]. However, Lee et al. revealed that berberine increased AMPK phosphorylation, but it did not activate p38 [10]. Several groups investigated the effect of berberine on glucose transporters and their conclusions remain controversial. Two groups also reported that berberine was able to stimulate GLUT4 translocation $[10,34]$, but this activity was not observed by other groups [10,22]. Repeated intravenous administration of tetrandrine $(1.0 \mathrm{mg} / \mathrm{kg})$ to STZ-diabetic rats for 3 days resulted in an increase in the mRNA and protein levels of the GLUT4 in soleus muscle, in addition to the lowering of plasma glucose [35].

Although, the exact mechanisms of berberine lipidmodulating effects are still unknown, we found that berberine could downregulate $\operatorname{PPAR} \gamma, \mathrm{PGC}-1$, and $\mathrm{C} / \mathrm{EBP} \alpha$ and upregulate $\operatorname{PPAR} \alpha$. The nuclear receptor PPAR $\gamma$ and members of the C/EBP family take important roles in adipogenesis [36]. The expression of both gluconeogenic and $\beta$-oxidation genes is further potentiated by the nuclear hormone receptor coactivator PGC-1, a target of cyclic AMP (cAMP) response

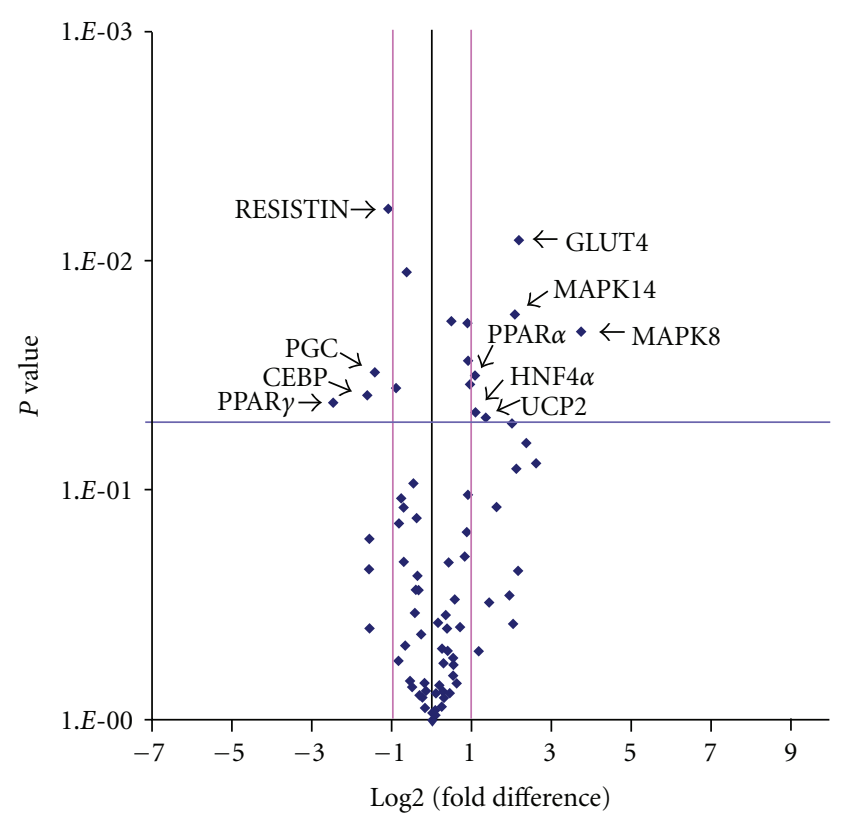

FIgUre 4: The Volcano Plot graphs of superarray. This graph shows that the $\log 2$ of the fold change in each gene's expression between berberine group and control group is versus its $P$ value from the $t$-test. The black line indicates fold changes of 1 . The pink lines indicate that the fold change in gene expression threshold is 2 . The blue line indicates that the $P$ value of the $t$-test threshold is .05 . There were 10 genes which showed significantly different expression between berberine group and control group.

element binding (CREB), whose levels are increased during fasting and in diabetes $[37,38]$. Berberine could downregulate the expression of PPAR $\gamma$, PGC-1, and C/EBP levels, which may be the mechanism that prevented KKAy mice treated with berberine from gaining weight. Weight gain has been identified as a class effect of the thiazolidinediones (TZDs). A factor contributing to TZD-related weight gain is increased adipocyte differentiation. The antidiabetic effects of TZDs are mediated through PPAR $\gamma$, a positive regulator of adipocyte differentiation [39]. Activation of PPAR $\gamma$ triggers the production of smaller more insulin-sensitive adipocytes, predominantly in the subcutaneous adipose compartment, and is likely to contribute to the TZD-mediated weight gain that has been observed in both animal and human studies [40]. Obesity is a major risk factor for metabolic syndrome and T2DM. However, most antidiabetic drugs that are hypoglycemic also promote weight gain, alleviating one symptom of T2DM while aggravating a major risk factor that leads to T2DM. It is highly desirable to develop pharmaceuticals and treatments for T2DM that reduce blood glucose levels without inducing adipogenesis in patients. Many studies have showed that berberine inhibited the mRNA and protein levels of adipogenesis-related transcription factors PPAR $\gamma$ and $\mathrm{C} / \mathrm{EBP} \alpha[10,41,42]$. Two groups confirmed that berberine increased PPAR $\alpha / \delta$ expression in liver and skeletal muscle tissue of diabetic rats $[43,44]$. PPAR $\alpha$ governs the expression of numerous genes involved in a variety of metabolic processes and modulates lipoprotein metabolism in several ways. 


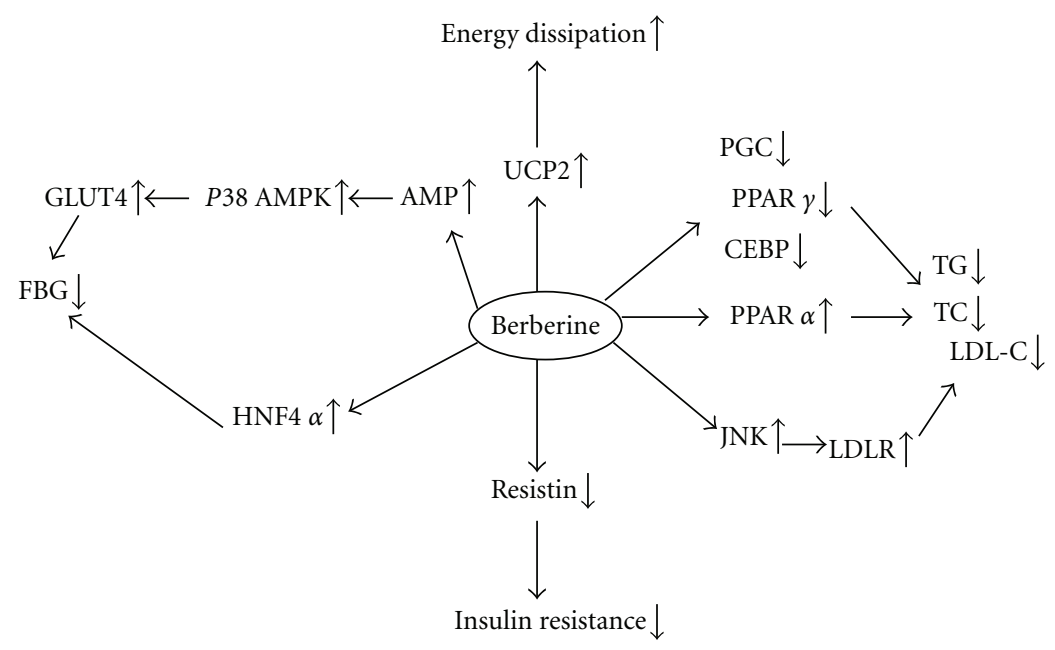

Figure 5: The mechanism of berberine moderating glucose and lipid metabolism.

TABLE 3: Effect of berberine on oral glucose tolerance in KKAy mice $\left(\mathrm{mmol} \cdot \mathrm{L}^{-1}\right)$.

\begin{tabular}{|c|c|c|c|c|c|c|}
\hline Group & $n$ & BG $0 \mathrm{~min}$ & BG $30 \mathrm{~min}$ & BG $60 \mathrm{~min}$ & BG $120 \mathrm{~min}$ & AUC \\
\hline Control & 8 & $24.74 \pm 8.89$ & $29.90 \pm 4.98$ & $22.10 \pm 10.67$ & $17.70 \pm 8.29$ & $45.45 \pm 10.81$ \\
\hline Berberine & 8 & $10.20 \pm 2.48^{* *}$ & $20.58 \pm 7.69^{* *}$ & $14.90 \pm 6.69^{*}$ & $11.08 \pm 6.36^{*}$ & $29.90 \pm 11.89^{*}$ \\
\hline
\end{tabular}

Values are means \pm SD.

*Indicates significantly different versus control $(P<.05)$; ** $(P<.01)$.

TABLE 4: Effect of berberine on FINS, HOMA-IR, and lipid metabolic parameters in KKAy mice.

\begin{tabular}{lccccccc}
\hline Group & $n$ & FINS $(\mu \mathrm{U} / \mathrm{mL})$ & HOMA-IR & TC $(\mathrm{mmol} / \mathrm{L})$ & TG $(\mathrm{mmol} / \mathrm{L})$ & HDL-c $(\mathrm{mmol} / \mathrm{L})$ & $\mathrm{LDL}-\mathrm{c}(\mathrm{mmol} / \mathrm{L})$ \\
\hline Control & 8 & $17.15 \pm 7.74$ & $13.45 \pm 5.07$ & $5.20 \pm 1.93$ & $1.29 \pm 0.40$ & $3.66 \pm 0.95$ & $0.86 \pm 0.20$ \\
Berberine & 8 & $6.80 \pm 3.36^{*}$ & $3.05 \pm 1.45^{*}$ & $3.03 \pm 0.69^{*}$ & $0.75 \pm 0.25^{*}$ & $4.00 \pm 1.12$ & $0.80 \pm 0.12$ \\
\hline
\end{tabular}

Values are means \pm SD

*Indicates significantly different versus control $(P<0.05)$.

TABLE 5: The genes that expressed differently between berberine group and control group (fold change $\geqslant 2.0, P<.05$ ).

\begin{tabular}{|c|c|c|c|c|}
\hline Gene name & Description & GeneBank ID & Fold change & $P$ value $t$-test \\
\hline SLC2A4 & Solute carrier family 2(GLUT4) & NM_009204 & 4.48 & .0081 \\
\hline MAPK8 & Mitogen-activated protein kinase 8(JNK) & NM_016700 & 13.15 & .0203 \\
\hline MAPK14 & Mitogen-activated protein kinase 14 & NM_011951 & 4.17 & .0171 \\
\hline $\operatorname{PPAR} \alpha$ & Peroxisome proliferator-activated receptor alpha & NM_011144 & 2.10 & .0316 \\
\hline $\operatorname{PPAR} \gamma$ & Peroxisome proliferator-activated receptor gamma & NM_011146 & -5.51 & .0412 \\
\hline CEBP & CCAAT/enhancer-binding protein, alpha & NM_011144 & -3.06 & .0385 \\
\hline $\operatorname{PPAR} \gamma \mathrm{C} 1 \alpha$ & $\begin{array}{l}\text { Peroxisome proliferative-activated receptor, gamma, } \\
\text { coactivator } 1 \text { alpha(PGC) }\end{array}$ & NM_008904 & -2.70 & .0306 \\
\hline UCP2 & Uncoupling protein 2 & NM_011671 & 2.55 & .0480 \\
\hline RETN & RESISTIN & NM_022984 & -2.12 & .0059 \\
\hline $\mathrm{HNF} 4 \alpha$ & Hepatic nuclear factor 4, alpha & NM_008261 & 2.12 & .0455 \\
\hline
\end{tabular}


Activation of PPAR $\alpha$ results in a reduction of plasma TG levels, which is achieved by induction of genes that decrease the availability of TG for hepatic VLDL secretion and induction of genes that promote lipoprotein lipase-mediated lipolysis of TG-rich plasma lipoproteins [45]. Upregulation of PPAR $\alpha$ may be the mechanism of the action of berberine in moderating lipid metabolism, just as in fibrate.

We also found that berberine could upregulate MAPK8 (JNK1). Berberine could increase the transcriptional activity of LDLR promoter and it involved the JNK pathway, reducing serum LDL [11]. Oral administration of berberine in 32 hypercholesterolemic patients for 3 months reduced serum cholesterol by $29 \%$, triglycerides by $35 \%$, and LDLcholesterol by $25 \%$ [19]. Treatment of hyperlipidemic hamsters with berberine reduced serum cholesterol by $40 \%$ and LDL-cholesterol by $42 \%$, with a 3.5 -fold increase in hepatic LDLR mRNA, and a 2.6-fold increase in hepatic LDLR protein [19]. Using human hepatoma cells, berberine upregulated LDLR expression independent of sterol regulatory element binding proteins but was dependent on ERK activation [46]. Although we found no evidence that berberine can reduce serum LDL, the upregulation of MAPK is a possible factor in the berberine lipid-regulating mechanism.

Moreover, berberine could upregulate UCP2. UCP2, which is found in many tissues, affects body weight gain, resting metabolic rates and food intake, which are all involved in energy balance [47]. In adipocytes and skeletal muscle tissue, UCP2 expression appears to increase following high-fat feeding. An increase in UCP2 levels might be expected to increase energy expenditure and decrease the risk of obesity. In skeletal muscle, the expression of UCP2 mRNA was significantly increased by berberine [10]. We speculated that bererine could upregulate UCP2, increase energy dissipation, and decrease the risk of obesity.

Also, we revealed that berberine could downregulate resistin. Resistin, as an adipocyte-secreted factor (ADSF), is a mouse protein with potential roles in insulin resistance and adipocyte differentiation. The TZD drug rosiglitazone can reduce resistin expression to improve insulin resistance. Ding et al. found that the effects of berberine in moderating lipids, as well as its contribution to reducing leptin and resistin, were closely correlated with its effects in ameliorating insulin resistance [48].

Additionally, berberine upregulated hepatic nuclear factor $4 \alpha(\mathrm{HNF} 4 \alpha)$. HNF $4 \alpha$ is a key regulator of a number of genes involved in glucose, cholesterol, and fatty acid metabolism [49]. The mRNA and protein expression of HNF- $4 \alpha$ were decreased in fructose-fed rats, but berberine promoted its expression [50]. Figure 5 shows the mechanism of berberine in moderating glucose and lipid metabolism.

In summary, our studies provide evidence that berberine reduces FBG and FINS and ameliorates insulin sensitivity and secretion in KKAy mice. Moreover, berberine reduces serum TC and TG and thereby regulates lipid metabolism. Berberine, in our study and others, caused changes in the expression of many genes that are involved in AMPKp38 MAPK-GLUT4, JNK pathway, and $\operatorname{PPAR} \alpha$ pathway.
These results provide molecular information for further investigation of the mechanisms by which berberine moderates glucose and lipid metabolism. Furthermore, these results could be important in devising mechanism-based and targeted therapeutic strategies for diabetes and lipid disorders. Further studies (i.e., real-time quantitative PCR) are planned to validate the gene expression profile changes observed with the $\mathrm{RT}^{2}$ Profiler PCR array.

\section{Acknowledgment}

This work was founded by Grants from the foundation of Peking Union Medical College Hospital (no. 2006119).

\section{References}

[1] R. M. O’Brien and D. K. Granner, "Regulation of gene expression by insulin,” Physiological Reviews, vol. 76, no. 4, pp. 1109-1161, 1996.

[2] P. A. Kiberstis, "A surfeit of suspects," Science, vol. 307, no. 5708, p. 369, 2005.

[3] Y. X. Ni, "Therapeutic effect of berberine on 60 patients with type II diabetes mellitus and experimental research," Zhong Xi Yi Jie He Za Zhi, vol. 8, no. 12, pp. 711-707, 1988 (Chinese).

[4] J. Yin, H. Xing, and J. Ye, "Efficacy of berberine in patients with type 2 diabetes mellitus," Metabolism, vol. 57, no. 5, pp. 712-717, 2008.

[5] A. F. G. Cicero, L. C. Rovati, and I. Setnikar, "Eulipidemic effects of berberine administered alone or in combination with other natural cholesterol-lowering agents: a single-blind clinical investigation," Arzneimittel-Forschung, vol. 57, no. 1, pp. 26-30, 2007.

[6] B. Lee, C. H. Yang, D.-H. Hahm et al., "Inhibitory effects of Coptidis rhizoma and berberine on cocaine-induced sensitization," Evidence-Based Complementary and Alternative Medicine, vol. 6, no. 1, pp. 85-90, 2009.

[7] L. Zhou, Y. Yang, X. Wang et al., "Berberine stimulates glucose transport through a mechanism distinct from insulin," Metabolism, vol. 56, no. 3, pp. 405-412, 2007.

[8] J. Yin, Z. Gao, D. Liu, Z. Liu, and J. Ye, "Berberine improves glucose metabolism through induction of glycolysis," American Journal of Physiology, vol. 294, no. 1, pp. E148-E156, 2008.

[9] N. Turner, J.-Y. Li, A. Gosby et al., "Berberine and its more biologically available derivative, dihydroberberine, inhibit mitochondrial respiratory complex I: a mechanism for the action of berberine to activate amp-activated protein kinase and improve insulin action," Diabetes, vol. 57, no. 5, pp. 14141418, 2008.

[10] Y. S. Lee, W. S. Kim, K. H. Kim et al., "Berberine, a natural plant product, activates AMP-activated protein kinase with beneficial metabolic effects in diabetic and insulin-resistant states," Diabetes, vol. 55, no. 8, pp. 2256-2264, 2006.

[11] S. Lee, H.-J. Lim, J.-H. Park, K.-S. Lee, Y. Jang, and H.-Y. Park, "Berberine-induced LDLR up-regulation involves JNK pathway," Biochemical and Biophysical Research Communications, vol. 362, no. 4, pp. 853-857, 2007.

[12] A. G. Smith and G. E. O. Muscat, "Skeletal muscle and nuclear hormone receptors: implications for cardiovascular and metabolic disease," International Journal of Biochemistry and Cell Biology, vol. 37, no. 10, pp. 2047-2063, 2005. 
[13] P. Chavan, K. Joshi, and B. Patwardhan, "DNA microarrays in herbal drug research," Evidence-Based Complementary and Alternative Medicine, vol. 3, no. 4, pp. 447-457, 2006.

[14] K. J. Livak and T. D. Schmittgen, "Analysis of relative gene expression data using real-time quantitative PCR and the 2- $\Delta \Delta$ CT method," Methods, vol. 25, no. 4, pp. 402-408, 2001.

[15] J. Yin, R. Hu, M. Chen et al., "Effects of berberine on glucose metabolism in vitro," Metabolism, vol. 51, no. 11, pp. 14391443, 2002.

[16] L.-Q. Tang, W. Wei, L.-M. Chen, and S. Liu, "Effects of berberine on diabetes induced by alloxan and a high-fat/highcholesterol diet in rats," Journal of Ethnopharmacology, vol. 108, no. 1, pp. 109-115, 2006.

[17] C. R. Gao, J. Q. Zhang, and Q. L. Huang, "Experimental study on berberin raised insulin sensitivity in insulin resistance rat models," Zhongguo Zhong Xi Yi Jie He Za Zhi, vol. 17, no. 3, pp. 162-164, 1997 (Chinese).

[18] J.-M. Brusq, N. Ancellin, P. Grondin et al., "Inhibition of lipid synthesis through activation of AMP kinase: an additional mechanism for the hypolipidemic effects of berberine," Journal of Lipid Research, vol. 47, no. 6, pp. 1281-1288, 2006.

[19] W. Kong, J. Wei, P. Abidi et al., "Berberine is a novel cholesterol-lowering drug working through a unique mechanism distinct from statins," Nature Medicine, vol. 10, no. 12, pp. 1344-1351, 2004.

[20] P. R. Shepherd, D. J. Withers, and K. Siddle, "Phosphoinositide 3-kinase: the key switch mechanism in insulin signalling," Biochemical Journal, vol. 333, no. 3, pp. 471-490, 1998.

[21] Z. Cheng, T. Pang, M. Gu et al., "Berberine-stimulated glucose uptake in L6 myotubes involves both AMPK and p38 MAPK," Biochimica et Biophysica Acta, vol. 1760, no. 11, pp. 1682-1689, 2006.

[22] S. H. Kim, E.-J. Shin, E.-D. Kim, T. Bayaraa, S. C. Frost, and C.-K. Hyun, "Berberine activates GLUT1-mediated glucose uptake in 3T3-L1 adipocytes," Biological and Pharmaceutical Bulletin, vol. 30, no. 11, pp. 2120-2125, 2007.

[23] Z. Gao, D. Hwang, F. Bataille et al., "Serine phosphorylation of insulin receptor substrate 1 by inhibitor $\kappa \mathrm{B}$ kinase complex," Journal of Biological Chemistry, vol. 277, no. 50, pp. 4811548121, 2002.

[24] M. R. Calera, G. Vallega, and P. F. Pilch, "Dynamics of proteintyrosine phosphatases in rat adipocytes," Journal of Biological Chemistry, vol. 275, no. 9, pp. 6308-6312, 2000.

[25] B. J. Goldstein, A. Bittner-Kowalczyk, M. F. White, and M. Harbeck, "Tyrosine dephosphorylation and deactivation of insulin receptor substrate- 1 by protein-tyrosine phosphatase 1B. Possible facilitation by the formation of a ternary complex with the GRB2 adaptor protein," Journal of Biological Chemistry, vol. 275, no. 6, pp. 4283-4289, 2000.

[26] J. L. Bento, N. D. Palmer, J. C. Mychaleckyj et al., "Association of protein tyrosine phosphatase $1 \mathrm{~B}$ gene polymorphisms with type 2 diabetes," Diabetes, vol. 53, no. 11, pp. 3007-3012, 2004.

[27] S. Kipfer-Coudreau, D. Eberlé, M. Sahbatou et al., "Single nucleotide polymorphisms of protein tyrosine phosphatase $1 \mathrm{~B}$ gene are associated with obesity in morbidly obese French subjects," Diabetologia, vol. 47, no. 7, pp. 1278-1284, 2004.

[28] R. Di Paola, L. Frittitta, G. Miscio et al., "A variation in 3' utr of hptplb increases specific gene expression and associates with insulin resistance," American Journal of Human Genetics, vol. 70, no. 3, pp. 806-812, 2002.
[29] J. M. Zabolotny, F. G. Haj, Y.-B. Kim et al., "Transgenic overexpression of protein-tyrosine phosphatase $1 \mathrm{~B}$ in muscle causes insulin resistance, but overexpression with leukocyte antigen-related phosphatase does not additively impair insulin action," Journal of Biological Chemistry, vol. 279, no. 23, pp. 24844-24851, 2004.

[30] R. Somwar, D. Y. Kim, G. Sweeney et al., "GLUT4 translocation precedes the stimulation of glucose uptake by insulin in muscle cells: potential activation of GLUT4 via p38 mitogenactivated protein kinase," Biochemical Journal, vol. 359, no. 3, pp. 639-649, 2001.

[31] X. Xi, J. Han, and J.-Z. Zhang, "Stimulation of glucose transport by AMP-activated protein kinase via activation of p38 mitogen-activated protein kinase," Journal of Biological Chemistry, vol. 276, no. 44, pp. 41029-41034, 2001.

[32] D. Konrad, R. Somwar, G. Sweeney et al., "The antihyperglycemic drug $\alpha$-lipoic acid stimulates glucose uptake via both GLUT4 translocation and GLUT4 activation: potential role of p38 mitogen-activated protein kinase in GLUT4 activation," Diabetes, vol. 50, no. 6, pp. 1464-1471, 2001.

[33] G. Zhou, R. Myers, Y. Li et al., "Role of AMP-activated protein kinase in mechanism of metformin action," Journal of Clinical Investigation, vol. 108, no. 8, pp. 1167-1174, 2001.

[34] B.-S. Ko, S. B. Choi, S. K. Park, J. S. Jang, Y. E. Kim, and S. Park, "Insulin sensitizing and insulinotropic action of berberine from Cortidis Rhizoma," Biological and Pharmaceutical Bulletin, vol. 28, no. 8, pp. 1431-1437, 2005.

[35] J. H. Hsu, J. C. Wu, S. S. Liou, I. M. Liu, L. W. Huang, and J. T. Cheng, "Mediation of endogenous $\beta$-endorphin by tetrandrine to lower plasma glucose in streptozotocin-induced diabetic rats," Evidence-Based Complementary and Alternative Medicine, vol. 1, no. 2, pp. 193-201, 2004.

[36] E. D. Rosen and O. A. MacDougald, "Adipocyte differentiation from the inside out," Nature Reviews Molecular Cell Biology, vol. 7, no. 12, pp. 885-896, 2006.

[37] J. C. Yoon, P. Puigserver, G. Chen et al., "Control of hepatic gluconeogenesis through the transcriptional coaotivator PGC1," Nature, vol. 413, no. 6852, pp. 131-138, 2001.

[38] S. Herzig, F. Long, U. S. Jhala et al., "CREB regulates hepatic gluconeogenesis through the coactivator PGC-1," Nature, vol. 413, no. 6852, pp. 179-183, 2001.

[39] B. M. Spiegelman, "PPAR- $\gamma$ : adipogenic regulator and thiazolidinedione receptor," Diabetes, vol. 47, no. 4, pp. 507-514, 1998.

[40] F. M. A. C. Martens, F. L. J. Visseren, J. Lemay, E. J. P. De Koning, and T. J. Rabelink, "Metabolic and additional vascular effects of thiazolidinediones," Drugs, vol. 62, no. 10, pp. 14631480, 2002.

[41] C. Huang, Y. Zhang, Z. Gong et al., "Berberine inhibits 3T3L1 adipocyte differentiation through the PPAR $\gamma$ pathway," Biochemical and Biophysical Research Communications, vol. 348, no. 2, pp. 571-578, 2006.

[42] L. B. Zhou, M. D. Chen, X. Wang et al., "Effect of berberine on the differentiation of adipocyte," Zhonghua Yi Xue Za Zhi, vol. 83, no. 4, pp. 338-340, 2003 (Chinese).

[43] J. Y. Zhou, S. W. Zhou, K. B. Zhang et al., "Chronic effects of berberine on blood, liver glucolipid metabolism and liver PPARs expression in diabetic hyperlipidemic rats," Biological and Pharmaceutical Bulletin, vol. 31, no. 6, pp. 1169-1176, 2008.

[44] J. Y. Zhou and S. W. Zhou, "Effect of berberine on glucolipid metabolization in diabetic skeletal muscle and its mechanism," Journal of Chinese Pharmaceutical Sciences, vol. 16, no. 4, pp. 300-306, 2007. 
[45] C. Duval, M. Müller, and S. Kersten, "PPAR $\alpha$ and dyslipidemia," Biochimica et Biophysica Acta, vol. 1771, no. 8, pp. 961-971, 2007.

[46] S. A. Doggrell, "Berberine - a novel approach to cholesterol lowering," Expert Opinion on Investigational Drugs, vol. 14, no. 5, pp. 683-685, 2005.

[47] C. Fleury, M. Neverova, S. Collins et al., "Uncoupling protein2: a novel gene linked to obesity and hyperinsulinemia," Nature Genetics, vol. 15, no. 3, pp. 269-272, 1997.

[48] L. B. Ding, F. E. Lu, A. L. Ye, L. Xu, and K. Wang, "Effects of huanglian jiedu decoction on leptin and resistin in insulin resistive rats," Zhongguo Zhong Xi Yi Jie He Za Zhi, vol. 26, no. 3, pp. 232-235, 2006 (Chinese).

[49] L. Love-Gregory and M. A. Permutt, "HNF4A genetic variants: role in diabetes," Current Opinion in Clinical Nutrition and Metabolic Care, vol. 10, no. 4, pp. 397-402, 2007.

[50] Z. Gao, S. Leng, F. Lu, M. Xie, L. Xu, and K. Wang, "Effect of berberine on expression of hepatocyte nuclear factor- $4 \alpha$ in rats with fructose-induced insulin resistance," Journal of Huazhong University of Science and Technology-Medical Science, vol. 28, no. 3, pp. 261-265, 2008. 


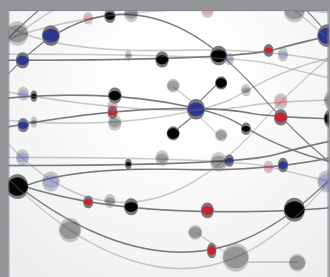

The Scientific World Journal
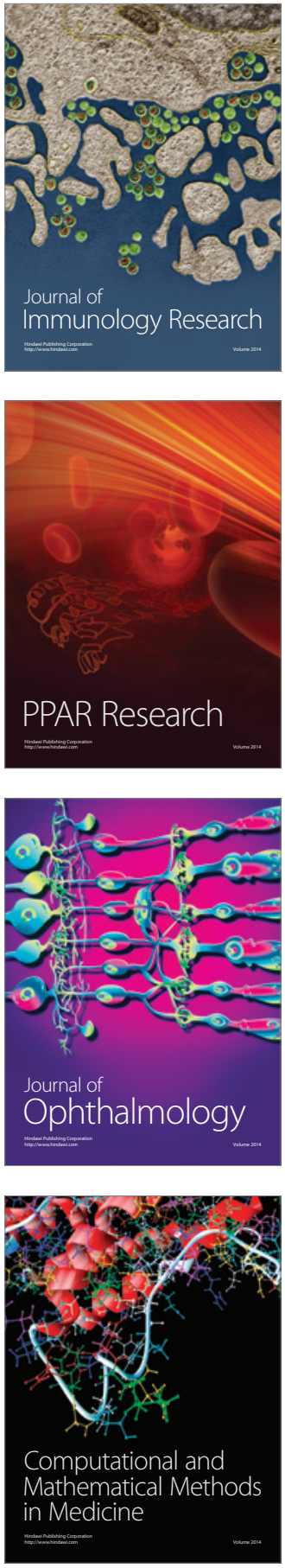

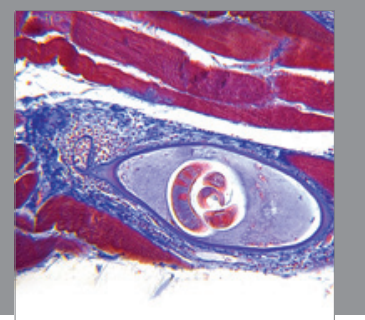

Gastroenterology

Research and Practice
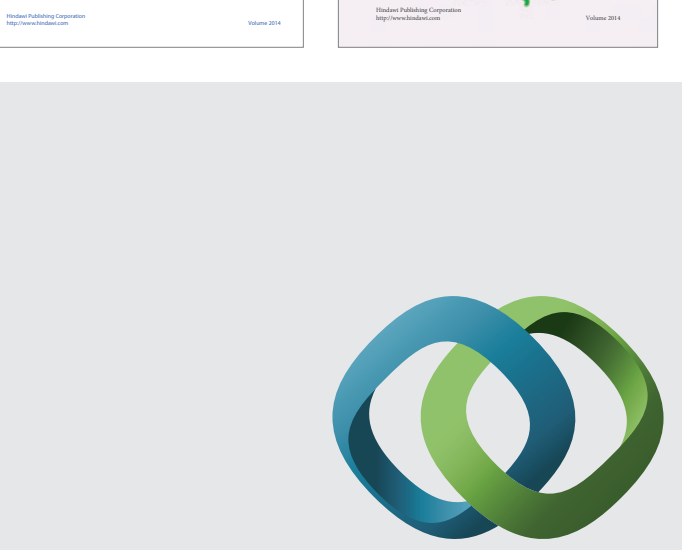

\section{Hindawi}

Submit your manuscripts at

http://www.hindawi.com
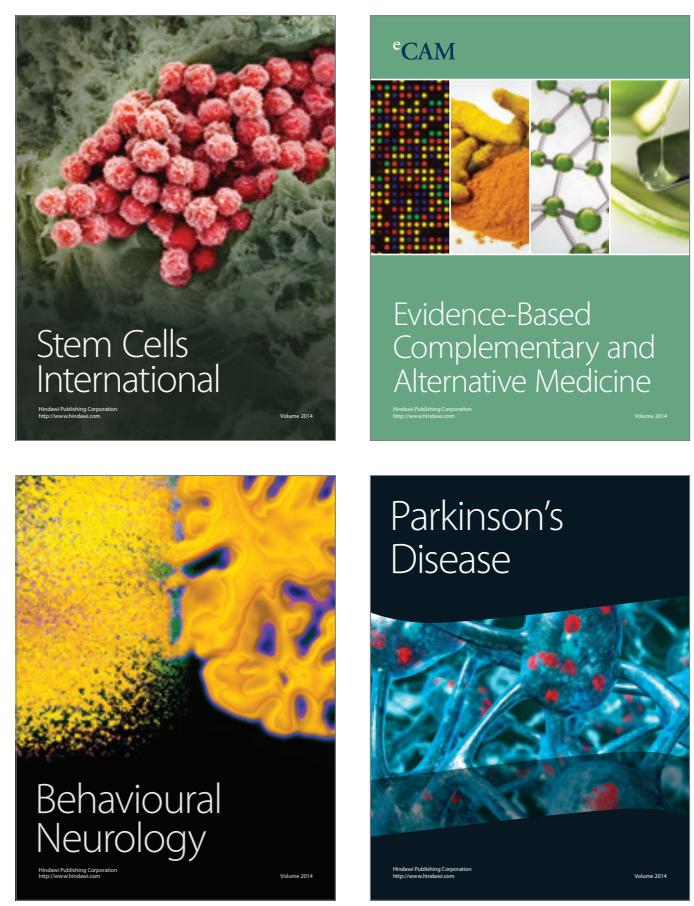

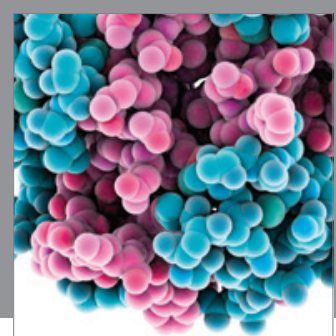

Journal of
Diabetes Research

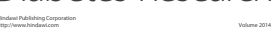

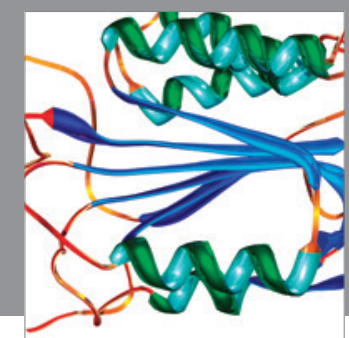

Disease Markers
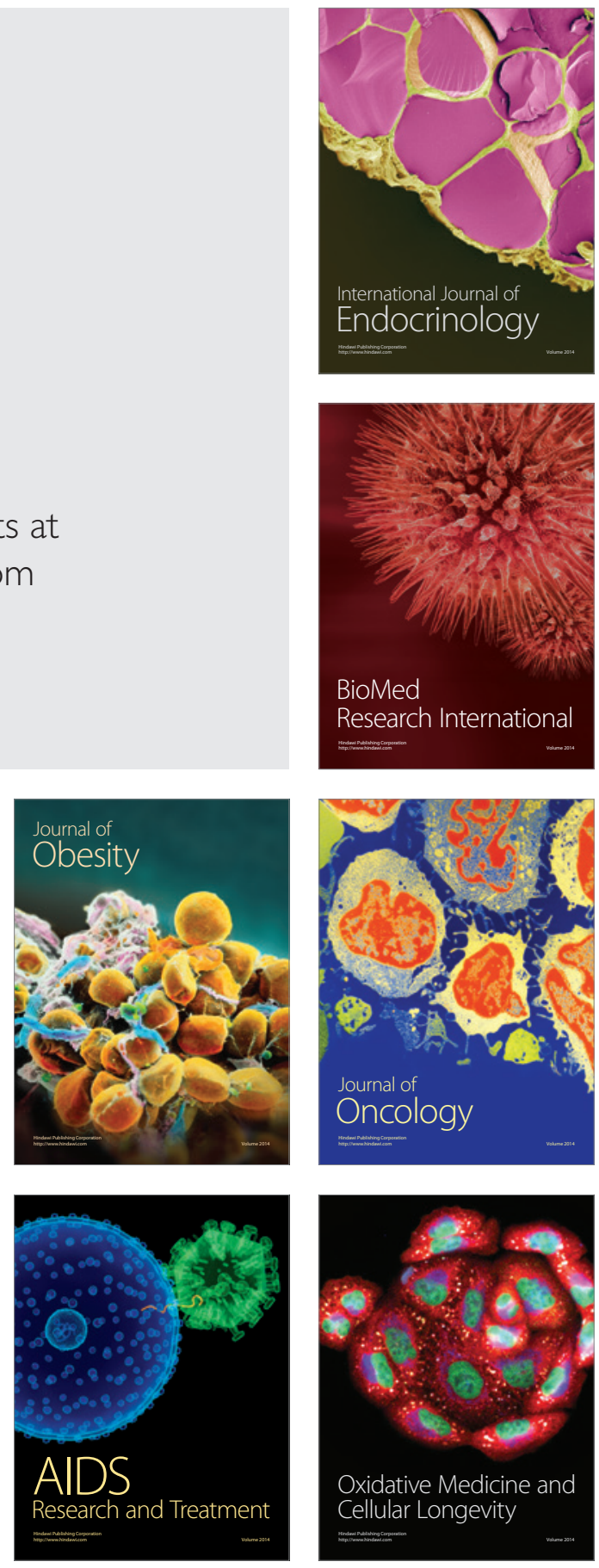